\title{
Characterization and Optimization of a Collinear Array of Circularly Polarized Side-Mounted Elements
}

\author{
Gerino P. Mappatao ${ }^{1}$, Pinky Tumaliuan-Jimenez ${ }^{2}$ \\ ${ }^{1}$ Department of Electronics and Communications Engineering, De La Salle University, Manila, Philippines \\ ${ }^{2}$ Department of Electronics Engineering, Cagayan State University, Tuguegarao City, Philippines
}

\begin{tabular}{l}
\hline \hline Article Info \\
\hline Article historys: \\
Received May 2, 2020 \\
Revised Sep 12, 2020 \\
Accepted Oct 2, 2020 \\
\hline
\end{tabular}

Keywords:

Circularly Polarized Antenna

FM Antenna

Collinear Array

Shunt-fed Slanted Dipole

Antenna Optimization

\begin{abstract}
This paper presents the radiation characteristics of a two-element collinear array of shunt-fed, slanted dipoles side-mounted to a metallic tower. It presents the radiation characteristics of the array as commonly implemented by FM broadcasters and suggests methods on how to optimize these characteristics such as the power gain, pattern circularity, side-lobe reduction, and horizontal-vertical polarization component ratio for the array to better conform to broadcast standards. By determining these characteristics using an advance antenna simulation software, optimization variables are identified, and their optimized values are determined. The optimized values lead to improved radiation characteristics of the array in conforming with broadcast requirements. Results show that adjusting the distance between the dipoles to less than one wavelength reduces the sidelobe of the array radiation pattern; varying the dipole physical specifications improves the polarization component ratio and the circularity of the radiation pattern. However, varying the dipole specifications also reduces the gain of the array. For applications requiring higher gains, other methods of realizing high collinear array gains are recommended.
\end{abstract}

Copyright $(\odot) 2020$ Institute of Advanced Engineering and Science. All rights reserved.

\section{Corresponding Author:}

Gerino P. Mappatao,

Department of Electronics and Communications Engineering,

De La Salle University,

2401 Taft Avenue, 0922 Malate, Manila, Philippines.

Email: gerino.mappatao@dlsu.edu.ph

\section{INTRODUCTION}

FM antenna systems are constructed by stacking identical elements or bays creating a collinear array. The number of bays being stacked depends on the antenna gain (and the Effective Radiated Power, ERP) that a broadcast station needs to cover its target area for a particular transmitter power. For a typical provincial application and with a transmitter power of anywhere between 1 and 5 kilowatts, a two-bay collinear antenna array is normal. It is enough to balance the ERP and the antenna height to cover a province. An antenna system with a higher gain and elevation maybe able to transmit over long distances but fails to cover the vicinity of the antenna because of reduced beamwidth. Generally, this paper aims to identify the limitations of this simple (simplest collinear array) antenna system that finds applications in medium-power provincial FM broadcast stations, and to suggest some methods to optimize its radiation performance. The array makes use of the patented Circularly Polarized Antenna (CPA), technically the same as the shunt-fed, slanted dipole.

Collinear arrays are used to realize higher antenna gains and are constructed by stacking identical bays. However, there are problems that need to be addressed with the use of the array: (1) presence of pattern nulls and the adverse effects of sidelobes (minor lobes); (2) effects of the metallic tower to the bays that tend to deviate the radiation pattern, and (3) the inherent non-compliant radiation characteristics of the CPA to broadcast standards. Stacking creates minor lobes and the number of which increases with the number of stacked elements [1-6]. One of the authors of this paper has a thorough discussion (including equations) on this topic in [1]. Minor lobes produce downward radiation and produce undesirable effects. Depending on the 
ERP, downward radiation may result to reception difficulties like blanketing as well as exposing the environment to excessive radiation [7-14]. Also, the presence of sidelobes produce pattern nulls between lobes that reduce the signal level within the service area of the station. Further, in the implementation of a collinear array, the bays are side-mounted in metallic towers. Previous papers show that the tower has effects on the radiation characteristics of the CPA [15-17], especially on the circularity of its radiation pattern. A single element side-mounted CPA produces radiation characteristics that do not conform to existing broadcast standards. It is therefore expected that, for a two-element CPA collinear array, the radiation characteristics are also deviated from the ideal values.

Regulatory bodies like the Federal Communications Commission of the United States and the Philippines' National Telecommunications Commission accept three kinds of proof-of-performance of FM broadcast antennas. These are the antenna characterization results from (1) actual tests and measurements in situ, (2) measurements in antenna test ranges, and (3) mathematical modelling including the results from simulation softwares. Using an advanced antenna simulation software, this paper aims to present the radiation characteristics of a two-bay collinear array using side-mounted CPA elements and suggests some optimized antenna configurations to improve the array's characteristics, particularly on the gain, pattern circularity, sidelobe reduction and horizontal-vertical polarization components ratio requirement.

\section{THE BAY}

Generally, the bay is developed to exhibit an omnidirectional radiation pattern on the horizontal plane; it could be horizontally $(\mathrm{H}-\mathrm{pol})$ or circularly $(\mathrm{C}-\mathrm{pol})$ polarized. For $\mathrm{C}$-pol bays, the vertically polarized (V-pol) component must not be greater than the $\mathrm{H}$-pol component. The bay used in this paper is the patented CPA. It is one of the most common antenna elements used in FM broadcast antenna systems. A lot of journal papers provide details of the CPA, especially on the physical dimensions and description of its construction and even its characterization in different situations and conditions [14-19]. The CPA or the slanted, shunt-fed dipole is illustrated in Figure 1 showing the isometric, top and front views. Two of this kind are used to form a collinear array in this paper. Its dimensions and other specifications are shown in Table 1. The wavelength that corresponds to a frequency in this paper is denoted by $\lambda$. Figure 1 and Table 1 are based on the presentations of [17] of the original patented CPA. In this paper, FEKO antenna simulation software is used in determining the radiation characteristics of the array, including the optimum values to produce the most desired radiation characteristics possible.

Based on the results presented in [17], with a tower diameter of 4 inches and $0.375 \lambda$ away from bay center, the CPA exhibits poor compliance to the required polarization component requirement, i.e. the V-pol component should not be greater than the H-pol component. Further, the radiation pattern circularity is affected by the presence of the metallic tower, especially the V-pol component. To improve bay performance, it is suggested that some bay dimensions and physical specifications must be adjusted.

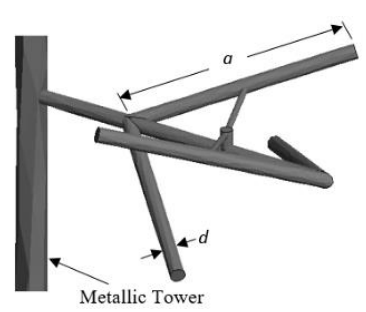

(a)

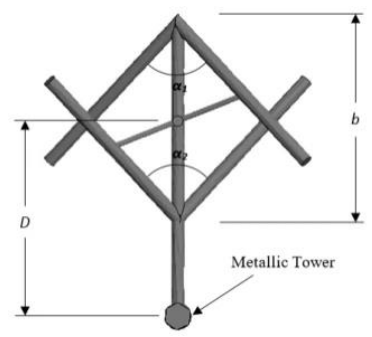

(b)

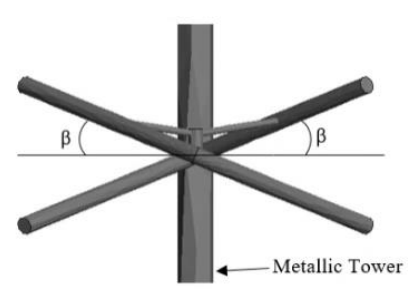

(c)

Figure 1. The CPA in (a) isometric view, (b) top view and (c) front view. The metallic tower length is two wavelengths long.

Table 1. Bay Dimensions and Physical Specifications

\begin{tabular}{lc}
\hline \multicolumn{1}{c}{ Dimension/ } & Value \\
\hline$b$ (boom length) & $1 / 4 \lambda$ \\
$d$ (Boom and arm diameter) & $\lambda / 120$ \\
$a$ (Arm length) & $1 / 4 \lambda$ \\
$\alpha_{1}$ (Dipole1 Arm offset angle) & $90^{0}$ \\
$\alpha_{2}$ (Dipole2 Arm offset angle) & $90^{0}$ \\
$\beta$ (Skew angle) & $22.5^{0}$ \\
\hline
\end{tabular}

\section{METHODOLOGY}


The set-up of the collinear array of two side-mounted CPA elements is shown in Figure 2. The radiation characteristics of the collinear array such as the gain, pattern circularity, side-lobe reduction and polarization components ratio will be presented, both for the initial and the optimised conditions. For the initial condition, the distance between bays, $\mathrm{C}$, is set at one wavelength, and each bay follows the dimensions and specifications shown in Table 1 . The tower-to-bay center distance, D, is initially set at $0.375 \lambda$. The set-up is simulated, and the radiation characteristics are then presented and analized. The metallic tower where the bays are mounted assumes a diameter size of 4 inches, the usual tower size used by broadcasters.

Based on published papers [1-2] [18-24], the gain and the side-lobe can be adjusted by varying the distance between the bays $(C)$. Also, the pattern circularity is altered by varying the tower distance from the bays (D), the skew angle ( $\beta$ ) and the dipole arm offset angle $(\alpha)$. Lastly, V-pol and H-pol relationship is adjusted by varying the skew angle. The optimization variables are, therefore, $\mathrm{D}, \mathrm{C}, \beta$ and $\alpha$. The optimal values of these variables are determined using the optimization tool of the software used.

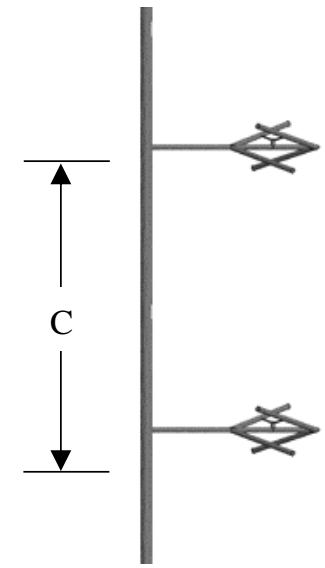

Figure 2. The two-element collinear array set-up

\section{RESULTS AND DISCUSSIONS}

The illustrations shown in Figure 3 are the radiation characteristics of the collinear array in the initial condition. The simulation was done with a working frequency of $97.4885 \mathrm{MHz}$. This is the center frequency of the FM band (88-108 MHz) in the Philippines and the United States. Figure 3(a) is the polar plot of the gain in $\mathrm{dBi}$ on the azimuth illustrating the circularity of the radiation pattern. From this point on, all gains are referred to the gain of an isotropic antenna. As shown, the circularity is $\pm 1.89 \mathrm{~dB}$, satisfying the required circularity of $\pm 2 \mathrm{~dB}$ for broadcast antennas. Note that the metallic tower is located at $180^{\circ}$ and has the effect of blocking the array radiation toward this direction. Figure 3(b) shows the polar plots of the power gains (total, V-pol and H-pol). This figure shows the performance of the collinear array to the H-pol over Vpol component ratio requirement. The $\mathrm{H}$-pol component is much smaller than the $\mathrm{V}$-pol component. The collinear array definite fails in this requirement, i.e. the $\mathrm{V}$-pol component must not be greater than the H-pol. This radiation characteristic needs improvement.

Figure 3(c) is the radiation pattern of the array in the vertical plane with the cutting plane in the $\mathrm{x}-\mathrm{Z}$ $\left(\varphi=0^{0}\right)$ plane. Figure 3(d) differs from Figure 3(c) only in the cutting plane, being in the $y-z\left(\varphi=90^{0}\right)$ plane. Figure 3(c) has relatively smaller sidelobes (downward radiation) compared to Figure 3(d). In the former, the larger sidelobe has a power gain of 0.86 at $55^{\circ}$ below the horizon (at $\theta=145^{\circ}$ ), while the latter has a sidelobe power gain of 1.01 also at $55^{\circ}$ below the horizon. At $55^{\circ}$ below the horizon, the sidelobes radiate power near the base of the tower (about 32 meters from the tower base for a 45 -meter tower) where the radiators are mounted. With the power gains in the sidelobes, a significant amount of power may produce blanketing and excessive radiation near the base of the antenna system. Reducing the amount of downward radiation due to the minor lobes is just proper. Also shown in Figure 3(c) and Figure 3(d), the beamwidths between halfpower points and first null are $30^{\circ}$ and below. Although these beamwidth values can produce a good coverage in most applications, a higher beamwidth value is better. Figure 3(e) shows the highest power gain (expessed as a power ratio) of the array over the whole FM band. Along the direction of the x-axis (phi, $\varphi=$ $0^{0}$ ), there is significant variation of the gain over the $20 \mathrm{MHz}$ FM band. This is because of the effect of the metallic tower on the gain of the array. If the bandwidth of the array is to be improved, tower effect must be minimized. Along the $y$-axis (phi, $\varphi=90^{\circ}$ ), the power gain is relatively constant. Note that the $y$-axis is perpendicular to the direction toward the tower; there is some sort of symmetry making the gain relatively the same. At the center frequency, the maximum power gain is $4.343(6.378 \mathrm{dBi})$ and occurs at $70^{\circ}$ on the horizontal plane. 


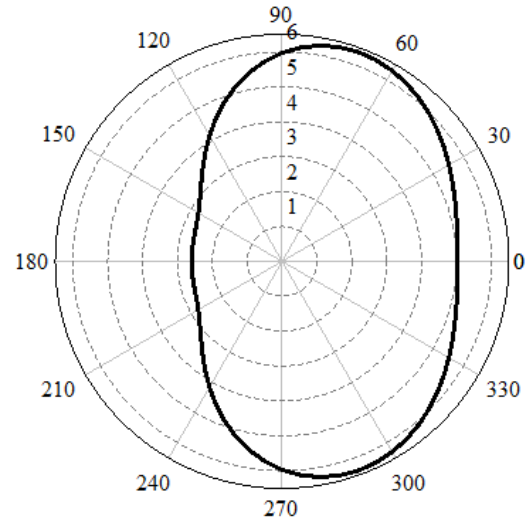

(a)

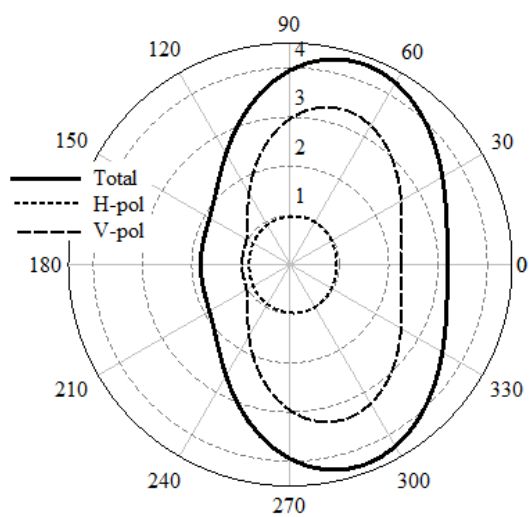

(b)

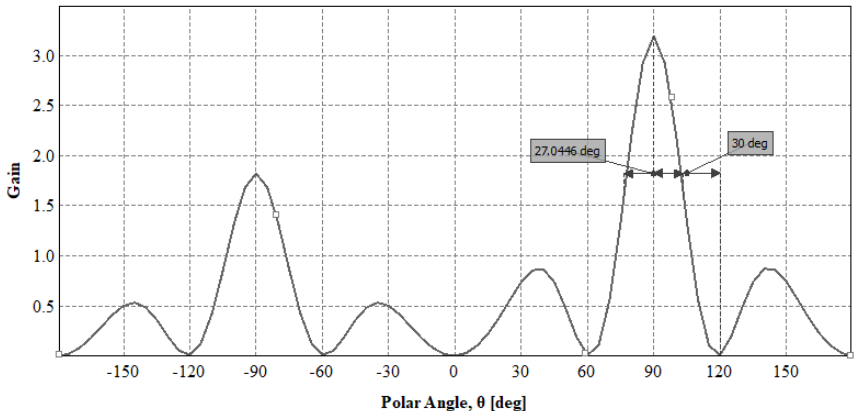

(c)

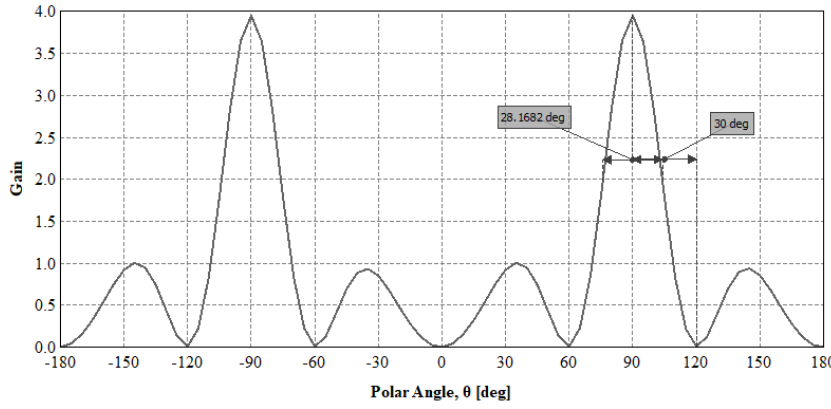

(d)

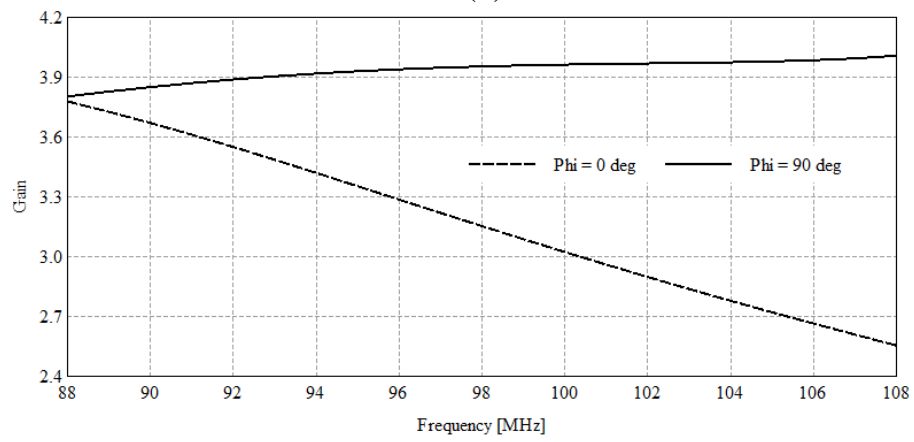

(e)

Figure 3. The radiation characteristics of the collinear array at the initial conditions: (a) gain showing the circularity of the radiation pattern, (b) power gains comparing the H-pol and the V-pol components, (c) radiation pattern on the $\mathrm{X}-\mathrm{Z}$ vertical plane, (d) radiation pattern on the $\mathrm{y}-\mathrm{z}$ vertical plane, (e) gain over the FM band

From the results of the characterization of the array in the initial condition, it is suggested that (a) the downward radiation near the tower base must be reduced, (b) the V-pol component must be reduced and make the H-pol component greater, and (c) the gain over the entire FM band is made relatively constant to improve the bandwidth of the array. 
With the use of the optimization variables to address the problems and obtaining the desired radiation characteristics described above, the results of the optimization suggest that the optimum values of the variables are the following: skew angle $(\beta)$ is $13^{\circ}$, offset angle $(\alpha)$ is $80^{\circ}$, tower-to-bay center distance (D) is at least one-half of a wavelength and bay distance of 0.7 of a wavelength. Figure 4(a) to Figure(d) illustrate the radiation characteristics of the optimized collinear array antenna simulated at the center of the FM band. The circularity of the optimized array is illustrated in Figure 4(a) using the power gain (dBi) polar plot of the array on the azimuth. The circularity is $\pm 1.88 \mathrm{~dB}$, which is slightly better than the circularity at initial condition. The H-pol/V-pol relationship is shown in Figure 4(b). The figure is a polar plot of the total power gain and its components on the horizontal plane. The H-pol at all angles is higher than its V-pol counterpart, thereby satisfying the $\mathrm{H}-$ pol/ $\mathrm{V}$-pol ratio requirement. A value of the skew angle lower that is than $13^{\circ}$ can further improve the H-pol/V-pol ratio, however, this decreases the gain of the antenna array. Figure 4(c) and Figure 4(d) are the radiation patterns of the array on the vertical plane. The cutting plane in the former is at the $\mathrm{X}-\mathrm{z}\left(\varphi=0^{0}\right)$ plane, while in the latter, the cutting plane is at the $\mathrm{y}-\mathrm{z}\left(\varphi=90^{\circ}\right)$ plane. At these planes, the highest downward radiation has a power gain of 0.11997 at about $65^{\circ}$ below the horizon $\left(\right.$ at $\left.\theta=155^{\circ}\right)$; there is a significant decrease in the gain compared to the initial condition. Also, the optimized array has a substantial increase in the beamwidth of the radiation pattern compared to the pattern beamwidth produced in the initial condition. With this beamwidth, greater antenna height is possible that allows coverage over long distances but not necessarily missing the immediate vicinity of the array. Further, a bay distance of $0.75 \lambda$ can make the downward radiation to a minimum, but this also decreases the gain. Figure 4(e) shows the maximum power gain of the array over the entire FM band along the $y$ - and $x$-axes. Compared to the initial condition, the gain of the optimized array has relatively flat response over the entire FM broadcast band. At $97.4885 \mathrm{MHz}$, the maximum power gain of the optimized array is $4.329(6.364 \mathrm{dBi})$ at $90^{\circ}$ on the azimuth, which is slightly below the value at the initial condition.

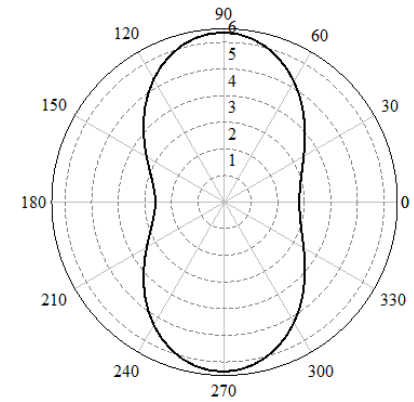

(a)

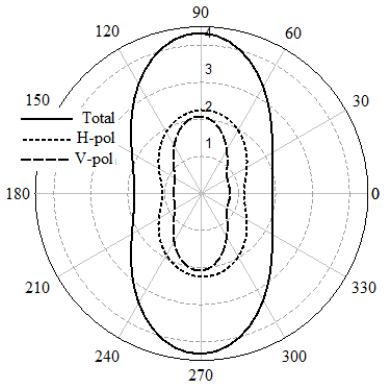

(b)

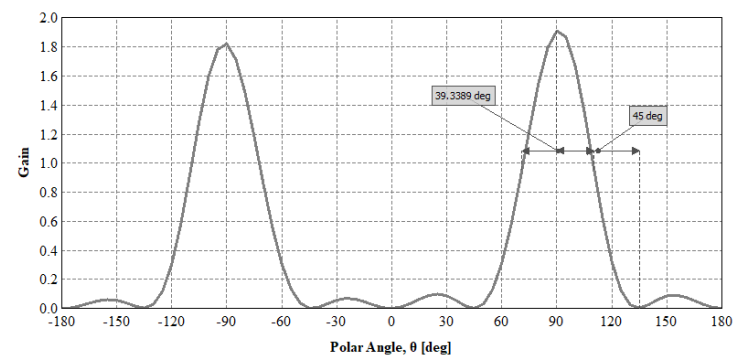

(c)

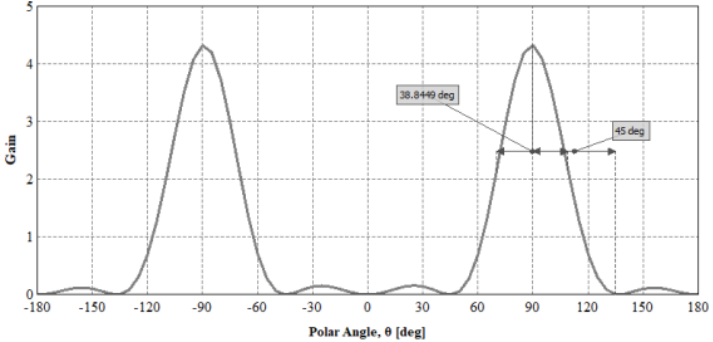

(d) 


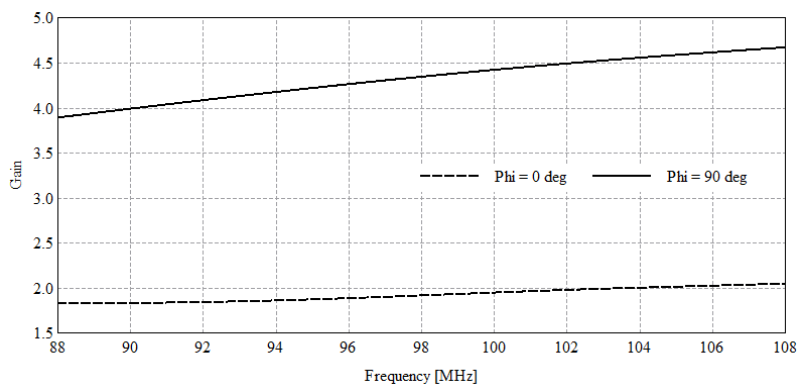

(e)

Figure 4. Radiation characteristics of the optimized collinear array: (a) gain showing the circularity of the radiation pattern, (b) power gains comparing the $\mathrm{H}$-pol and the $\mathrm{V}$-pol components, (c) radiation pattern on the $x-z$ vertical plane, (d) radiation pattern on the $y-z$ vertical plane, (e) gain over the FM band

Though the optimized array is intended for FM broadcast applications, it is possible that it can find applications in other communication systems such as the ones discussed in [25-30]. When the optimized array is used in such systems, its advantages and limitations must be considered, and proper frequency scaling is necessary.

\section{CONCLUSION}

The radiation characteristics of a two-element CPA collinear array arranged in the most common way is presented as the initial condition in this paper. While it is true that the array produces a pattern with the required circularity and gain, little that we know that it is basically a V-pol radiator, i.e. the V-pol component of the radiation pattern is higher than the $\mathrm{H}$-pol component. The downward radiation can produce excessive radiation near the tower base and can pose adverse effects, therefore, it needs to be reduced. Adjusting the distance between bays is effective in sidelobe reduction; inter-bay distance of $0.7 \lambda$ is suggested. The skew angle is effective in increasing the H-pol component over the V-pol component, though a reduction of the total power gain is observed in so doing. Decreasing the offset angle improves the circularity but it also decreases the gain of the array. Generally, in complying with broadcast requirements, especially on circularity and polarization ratio, the gain is reduced. However, with proper balance, compliance and fair power gain value can be achieved. Since every responsible broadcaster is expected to conform to all broadcast requirements and standards, it is suggested that, for future directives, the optimum variable values described above are to be observed. Also, other ways in attaining higher gains to compensate for the gain reductions must be explored. A four-bay collinear antenna is recommended, however, its compliance to all broadcast requirements is also necessary.

\section{ACKNOWLEDGEMENTS}

The author would like to thank University Research Coordination Office (URCO) and DLSU Science foundation for the realization of this paper.

\section{REFERENCES}

[1] G.P. Mappatao, "Reducing the downward radiation of slanted dipole array," ISIEA 2010 - 2010 IEEE Symposium on Industrial Electronics and Applications, 2010, pp. 290-294.

[2] G.P. Mappatao, "Radiation pattern shaping for FM broadcast-optimizing coverage," ISIEA 2010 - 2010 IEEE Symposium on Industrial Electronics and Applications, 2010, pp. 222-225.

[3] N. Mukit, M. Islam, M. Habaebi, Z. Alam, K. Abdullah, N. Malek, R. Nibir, N. Adnan, E. Osman, "Designing large-scale antenna array using sub-array," Bulletin of Electrical Engineering and Informatics, vol. 8, no. 3, pp. 906-915, 2019.

[4] A. Taybi, A. Tajmouati, J. Zbitou, L. El Abdellaoui, M. Latrach, A. Errkik, "A new configuration of patch antenna array for rectenna array applications," TELKOMNIKA Telecommunication, Computing, Electronics and Control, vol. 17, no. 5, pp. 2186-2193, 2019.

[5] R. Nibir, I. Rafiqul, M. Habaebi, S. Yasmin, N. Mukit, S. Rafiq, A. O, "Multiband antenna using stacked series array for Ka-Band application," Bulletin of Electrical Engineering and Informatics, vol. 8, no. 3, pp. 1004-1013, 2019.

[6] N. Malek, O. Khalifa, Z. Abidin, S. Mohamad, N. Rahman, "Beam Steering using the Active Element Pattern of Antenna Array," TELKOMNIKA Telecommunication, Computing, Electronics and Control, vol. 16, no. 4, pp. $1542-1550,2018$. 
[7] A. Mumin, R. Alias, J. Abdullah, S. Dahlan, J. Ali, "Assessment of Electromagnetic Absorption towards Human Head Using Specific Absorption Rate,” Bulletin of Electrical Engineering and Informatics, vol. 7, no. 4, pp. 657$664,2018$.

[8] F. Malek, K. A. Rani, H. A. Rahim, and M. H. Omar, "Effect of Short-Term Mobile Phone Base Station Exposure on Cognitive Performance, Body Temperature, Heart Rate and Blood Pressure of Malaysians," Nat. Publ. Gr., no. August, pp. 5-9, 2015.

[9] I. Fayed, "Electromagnetic Radiation and its effects on human beings: Survey and Environmental Recommendations," 15th Sci. Symp. Hajj, Umr. Madinah. Vol. 15, pp. 35-47, 2015.

[10] I. Fayed I, E. El-Din, "Safety Distance Calculations for Macrocell Mobile Base Station and on Site Radiation Measurements," Eur. Conf. Electr. Eng. Comput. Sci. 2017, pp. 449-453.

[11] A. H. Sallomi, "A theoretical approach for SAR calculation in human head exposed to RF signals," Journal of Engineering and Sustainable Development, vol. 16, no. 4, pp. 304-313, 2014.

[12] H. Y. Chen and T. H. Lin, "Simulations and measurements of electric fields emitted from a LTE base station in an urban area," Int. J. Antennas Propag., vol. 2014, 2014.

[13] IEEE, "IEEE Standard for Safety Levels with Respect to Human Exposure to Radio Frequency Electromagnetic Fields, 3 kHz to $300 \mathrm{GHz}$," in IEEE Std C95.1-2005 (Revision of IEEE Std C95.1-1991), vol., no., pp.1-238, 19 April 2006

[14] M. Khuzairi, HA Rahim, M. Abdulmalek, M. Warip, "Radio frequency radiation measurement for base tower station safety compliances: a case study in Pulau Pinang Malaysia," Bulletin of Electrical Engineering and Informatics, vol. 8, no. 1, pp. 150-157, 2019.

[15] G.P. Mappatao, "The compliance to broadcast standards of a side-mounted circularly polarized antenna," International Journal on Communications Antenna and Propagation, vol. 8, no. 3, pp. 240-247, 2018.

[16] G. Mappatao, "Compliance to broadcast standards of patented broadcast antennas: The circularly polarized Antenna," International Journal on Communications Antenna and Propagation, vol. 7, no. 2, pp. 154-161, 2017.

[17] G.P. Mappatao, "Radiation characteristics of patterns derived from shunt-fed slanted dipole linear arrays," International Journal on Communications Antenna and Propagation, vol. 6, no. 3, pp. 138-145, 2016.

[18] I.M.Z. Bautista, M.K. Orsos, M.A. Ribo, L. Castillo, G. Mappatao, "Development of a remote tending system for analog broadcast transmitters," Indonesian Journal of Electrical Engineering and Computer Science, vol. 15, no. 3, pp. 1474-1484, September 2019.

[19] G. Mappatao, "Reducing FM broadcast energy consumption using directional radiation pattern," ARPN Journal of Engineering and Applied Sciences, vol. 10, no. 15, pp. 6484-6490, 2015.

[20] I.M.Z. Bautista, M.K. Orsos, M.A. Ribo, L. Castillo, G. Mappatao, "Remote Tending of Modern Broadcast Transmitters," Indonesian Journal of Electrical Engineering and Computer Science, vol. 15, no. 3, pp. 1491-1500, September 2019.

[21] F.S. Caluyo, G.P. Mappatao, "Optimizing FM broadcast coverage using hybrid optimizations," International Journal on Communications Antenna and Propagation, vol. 1, no. 5, pp. 439-445, 2011.

[22] N. I. A. Ishak, Norhudah Seman , Tien Han Chua, "Antenna array design with rectangular ring slot for 5G technology," TELKOMNIKA Telecommunication, Computing, Electronics and Control, vol. 17, no. 5, pp. 22582267, 2019.

[23] F.S. Caluyo, G.P. Mappatao, "Azimuth radiation pattern shaping and control for FM broadcast," International Journal on Communications Antenna and Propagation, vol. 1, no. 3, pp. 284-289, 2011.

[24] Shubha Gupta, Poonam Kshirsagar, Biswajeet Mukherjee, "A Low-Profile Multilayer Cylindrical Segment Fractal Dielectric Resonator Antenna: Usage for Wideband Applications," IEEE Antennas and Propagation Magazine vol. 61, No. 4, pp. 55-63, 2019.

[25] D.M. Pangan, Q. Santos, G. Mappatao, C. Aragones, P.D. Elpa, "Scalable remote water monitoring system using radio frequency links," Lecture Notes in Electrical Engineering, vol. 379, pp. 13-23, 2016.

[26] H. Al Ibraheemi, M. M. A. Al Ibraheemi, "Wireless Communication System with Frequency Selective Channel OFDM Modulation Technique," TELKOMNIKA Telecommunication, Computing, Electronics and Control, vol. 18, no. 3, pp. 1203-1208, 2020.

[27] A. L. Imoize, S. D. Odeyemi, J. A. Adebisi, "Development of a Low-Cost Wireless Bee-Hive Temperature and Sound Monitoring System," Indonesian Journal of Electrical Engineering and Informatics, vol. 8, no. 3, 2020.

[28] B. Mukherjee, Pragati Patel, J. Mukherjee, "Hemispherical Dielectric Resonator Antenna Based on Apollonian Gasket of Circles-A Fractal Approach," IEEE Transactions on Antennas and Propagation, vol. 62, no. 1, pp. 40 47, 2014.

[29] Z. K. Hussein , H. J. Hadi , M. R. Abdul-Mutaleb , Y. S. Mezaal, "Low cost smart weather station using Arduino and ZigBee," TELKOMNIKA Telecommunication, Computing, Electronics and Control, vol. 18, no. 1, pp. 282-288, 2020.

[30] A. Z. Yonis, "Evolution of millimeter-wave communications toward next generation in wireless technologies," TELKOMNIKA Telecommunication, Computing, Electronics and Control, vol. 17, no. 6, pp. 3161-3167, 2019.

\section{BIOGRAPHY OF AUTHORS}




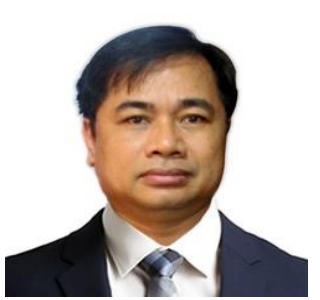

Gerino P. Mappatao was born in Tuao, Cagayan, Philippines. He received the B.S. degree in electronics and communications engineering (ECE) from Saint Louis University, Baguio City, Philippines in 1989, the M.S. and PhD degrees in ECE from De La Salle University-Manila, Philippines in 1998 and 2012, respectively.

$\mathrm{He}$ is currently an Associate Professor of Electronics Engineering at De La Salle University-Manila. He authored and co-authored papers in conference proceedings and journals on antennas, broadcast engineering, wireless communication, and image processing.

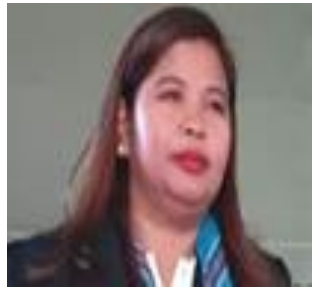

Pinky Tumaliuan-Jimenez is a licensed Professional Electronics Engineer in the Philippines. She received the B.S. degree in electronics and communications engineering (ECE) and the Master of Engineering major in Electronics Engineering from the University of Saint Louis, Tuguegarao City, Philippines.

She is currently an Assistant Professor of Electronics Engineering at Cagayan State University-Carig Campus, Tuguegarao City, Philippines. She is very active in the promotion and practice of electronics engineering in the Philippines. 